\title{
Evidencia Orientada al Paciente (EOP): Estrategias útiles en el tratamiento de la enuresis nocturna primaria en varones preadolescentes
}

\author{
Useful strategies in the management of primary nocturnal enuresis in preadolescent boys
}

Laura Fraguas*

\begin{abstract}
Resumen
La enuresis constituye un motivo de consulta frecuente en atención primaria, con una prevalencia general del 13 al $15 \%$ que varía en forma decreciente a mayor edad del niño. En la actualidad existen distintas estrategias de abordaje, desde intervenciones conductuales simples o complejas a intervenciones farmacológicas.

Partiendo de una viñeta clínica el autor se plantea si en niños varones preadolescentes que presentan enuresis nocturna primaria, la realización de algún tratamiento (comparada con la conducta expectante) se asocia a beneficios clínicos como un mayor número de noches secas.

Tras realizar una búsqueda bibliográfica y resumir una revisión sistemática pertinente a la pregunta formulada se concluye que las intervenciones conductuales simples como los refuerzos positivos, el entrenamiento vesical y despertar al niño durante la noche para que orine, pueden ser más efectivas que la conducta expectante, pero parecieran tener menor efectividad que el uso de las alarmas y los antidepresivos tricíclicos.
\end{abstract}

\begin{abstract}
Nocturnal enuresis is a frequent complaint in primary care, with an overall prevalence of 13 to $15 \%$ and decreasing occurrence as the child gets older. At present, there are different strategies of management, from simple or complex behavioral interventions to pharmacological interventions.

From a clinical vignette the author asks whether in preadolescent boys who have primary nocturnal enuresis, performing any treatment (compared with expectant management) is associated with clinical benefits such as an increased number of dry nights.

After conducting a literature search and summarizing a relevant systematic review, it concluded that simple behavioral interventions such as positive reinforcement, bladder training and waking the child at night to urinate, may be more effective than expectant management, but they seem to be less effective than the use of alarms and tricyclic antidepressants.
\end{abstract}

Fraguas L. Estrategias útiles en el tratamiento de la enuresis nocturna primaria en varones preadolescentes. Evid Act Pract Ambul. 2015;18(3):99100.Jul-Sep.

\section{Escenario clínico}

Una residente de medicina familiar de primer año atiende por primera vez a un niño de 11 años y a su madre que acuden a la consulta para realizar un control de salud. Al finalizar la consulta la madre le cuenta a la médica que el niño nunca logro controlar la micción por las noches y que presenta uno a dos episodios de enuresis nocturna por semana y refiere preocupación respecto a este tema. La médica decide citar nuevamente al paciente y a su madre para poder evaluar la enuresis primaria nocturna del niño y buscar herramientas para poder ayudarlo.

\section{Pregunta que generó el caso}

¿En niños varones de 11 años que presentan enuresis nocturna primaria (población), la realización de algún tratamiento (intervención) comparada con la conducta expectante (comparación) se asocia a beneficios clínicos como un mayor número de noches secas (resultado)?

\section{El problema}

La enuresis se ha definido como episodios repetidos de vaciamiento involuntario de la vejiga en niños mayores de cinco años ${ }^{1}$. Es un motivo de consulta frecuente en atención primaria que, en muchos casos, está motivada por el impacto psíquico que produce en el niño y en su familia ${ }^{2}$. Se la clasifica en primaria o secundaria, en diurna o nocturna y en monosintomática o no monosintomática (presentan en forma simultánea síntomas urinarios bajos) $)^{2}$. La enuresis primaria constituye el $80 \%$ de los casos y es aquella que (a diferencia de la secundaria) está presente en niños que nunca lograron controlar voluntariamente la micción por un periodo mayor a seis meses. La enuresis nocturna es tres veces más frecuente que la diurna ${ }^{2} y$ dicha entidad es tres veces más frecuente en varones que en mujeres ${ }^{3}$.

En la población general la prevalencia de enuresis nocturna es del 13 a 15\% y varía según la edad del paciente: en pacientes de cinco años la prevalencia es del $16 \%$, mientras que a los diez años es del $5 \%$ y en pacientes de 12 a 14 años la prevalencia es del 2 a $3 \%$. Estos datos sugieren que el trastorno resuelve espontáneamente con el paso de los años (tasa de curación anual del 15\%) $)^{1,5}$.

La enuresis primaria nocturna se produce por una alteración en la capacidad de la vejiga y la ausencia de despertar nocturno del niño al sentir la vejiga llena ${ }^{2}$. Existen diversas hipótesis sobre las posibles causas que generan este trastorno: un retraso de la maduración vesical, causas genéticas, vejiga funcionalmente pequeña, poliuria nocturna por una menor secreción nocturna de hormona antidiurética y alteraciones en el sueño ${ }^{2}$. En la actualidad existen diversos tratamientos: a) Intervenciones conductuales simples como disminuir el consumo de líquidos por la noche, despertar al niño durante la noche para que orine, reforzar positivamente las noches secas (calendario mensual con "stickers" en las noches secas), solicitar que el niño cambie las sábanas y las lleve al lavarropas, entrenamiento vesical ${ }^{\S}$, etc. ${ }^{2}$; b) Intervenciones conductuales complejas como el uso de alarmas que suenan cuando el niño se orina en la cama²; c) Intervenciones farmacológicas que incluyen el uso de anticolinérgicos (oxibutinina), antidepresivos triciclicos (imipramina, amitriptilina) y la desmopresina ${ }^{2}$.

\section{Estrategia de búsqueda}

Se realizó una búsqueda en MEDLINE con la siguiente estrategia: "Nocturnal Enuresis/therapy"[MeSH] limitando la búsqueda revisiones en los últimos diez años. La búsqueda arrojó varias citas de las cuales se seleccionó una que respondía la pregunta.

\section{Resumen de la evidencia}

Caldwell PHY, y col. Simple behavioural interventions for nocturnal enuresis in children. Cochrane Database of Systematic Reviews 2013;Issue 7. Art. No.: CD003637. DOI: 10.1002/14651858.CD003637.pub3.

* Servicio De Medicina Familiar y Comunitaria del Hospital Italiano de Buenos Aires. laura.fraguas@ hospitalitaliano.org.ar

$\S$ Se refieren a ejercicios realizados durante el día que intentan incrementar paulatinamente la capacidad vesical, retrasando el tiempo entre el deseo de orinar y la micción. 
Objetivo del estudio: Determinar los efectos de las intervenciones conductuales simples en niños con enuresis nocturna. Método de búsqueda: Se realizaron búsquedas en el Registro Especializado de Ensayos del Grupo Cochrane de incontinencia (Cochrane Incontinence Group) (hasta el 15 de diciembre 2011) y en las listas de referencia de artículos relevantes.

Criterios de selección: Se incluyeron ensayos clínicos aleatorizados o cuasi-aleatorizados de intervenciones conductuales simples comparadas con ningún tratamiento activo, otras intervenciones conductuales o con medicamentos, ya sea solas o en combinación para el tratamiento de enuresis nocturna en niños menores de 16 años.

Resultados principales: Se incluyeron 16 ensayos clínicos con 1.643 niños que recibieron intervenciones conductuales pero como las mismas fueron muy heterogéneas solo pudieron meta-analizarse aquellos estudios que evaluaron entrenamiento vesical comparado con terapia con alarmas nocturnas (Ver Tabla 1).
Las intervenciones conductuales simples comparadas con la ausencia de tratamiento activo fueron más efectivas, especialmente los refuerzos positivos en las noches secas con o sin despertar del niño para orinar. El entrenamiento vesical también fue más efectivo que la conducta expectante pero los estudios que lo sustentan son pequeños y de baja calidad metodológica. El uso de alarmas fue más efectivo que el entrenamiento vesical para el tratamiento de enuresis nocturna, pero no se encontraron beneficios adicionales cuando se combinaban ambas intervenciones. En un pequeño estudio se vio que la terapia cognitiva fue más efectiva que el refuerzo positivo.

El entrenamiento vesical es igual de efectivo que la desmopresina, oxibutinina o placebo en el tratamiento de la enuresis nocturna.

Los antidepresivos tricíclicos, amitriptilina y imipramina, fueron comparados con intervenciones conductuales simples y parecen ser más efectivos que estas últimas (solas o combinadas).

Tabla 1: Número de niños que no alcanzan 14 noches secas consecutivas al final del tratamiento.

\begin{tabular}{|c|c|c|c|}
\hline Intervención & Comparación & RR & IC $95 \%$ \\
\hline Refuerzos positivos & Control & 0,84 & 0,73 a 0,95 \\
\hline Refuerzo positivo + despertar & Control & 0,22 & 0,06 a 0,78 \\
\hline Despertar al niño & Control & 0,79 & 0,68 a 0,92 \\
\hline Entrenamiento vesical & Control & 0,85 & 0,63 a 1,15 \\
\hline Entrenamiento vesical & Alarma & 2,73 & 1,75 a 4,26 \\
\hline Entrenamiento vesical + alarma & Alarma sola & 1,77 & 0,50 a 6,23 \\
\hline Refuerzo positivo & Terapia cognitiva & 2,8 & 1,24 a 6,30 \\
\hline
\end{tabular}

Un Riesgo Relativo $(\mathrm{RR})<1$ implica una intervención más beneficiosa que el comparador

\section{Conclusiones}

Las intervenciones conductuales simples como los refuerzos positivos, el entrenamiento vesical y despertar al niño durante la noche para que orine, pueden ser más efectivas que la conducta expectante, pero parecieran tener menor efectividad que el uso de las alarmas y los antidepresivos tricíclicos, los que no están exentos de efectos adversos. De todas maneras hay que ser cautos al interpretar estos resultados ya que los ensayos clínicos incluidos en la revisión fueron pequeños y de pobre calidad metodológica.
En el caso del paciente presentado sería prudente averiguar el impacto del problema en el niño y el nivel de compromiso de los padres en el problema ya que mucha de las intervenciones conductuales requiere de un gran esfuerzo familiar para que sean llevadas a cabo. Teniendo esos datos y la evidencia aportada por la revisión comentada, se adecuaría la estrategia más efectiva al paciente y a su familia.

Recibido el 01/06/2015 y aceptado el 01/07/2015

\section{Referenciasbibliográficas}

1. Ramakrishnan K. Evaluation and treatment of enuresis. Am Fam Physician. 2008 Aug 15;78(4):489-96.

2. Renny MI. Enuresis. En: Voyer LE, y col. Pediatría. 3ra edición. Buenos Aires: Ed. Journal; 2011. p1135-1137.

3. Von Gontard A, y col. Clinical Behavioral problems in day-and night-wetting children. Pediatr Nephrol 1999;13:662.

4. Fergusson DM, y col. Factors related to the age of attainment of nocturnal bladder control: an 8-year longitudinal study. Pediatric 1986;78:884.

5. Pediatric Society of New Zealand. Nocturnal Enuresis "Bedwetting" (en línea). Wellington, Nueva Zelanda: Pediatric Society of New Zealand;2005. Disponible en URL: http://www.paediatrics.org.nz/files/guidelines/enuresisguidelinefinalendorsed.pdf (último acceso, septiembre 2015). 